\section{INCREASED INCIDENCE OF BRONCHOPULMONARY DYSPLASIA IN PRETERM INFANTS EXPOSED TO MATERNAL PREECLAMPSIA}

doi:10.1136/archdischild-2012-302724.0602

H Ozkan, M Cetinkaya, N Koksal. Uludağ University Medical Faculty, Bursa, Turkey

Objective The aims of the study were to determine the effect of preeclampsia on bronchopulmonary dysplasia (BPD) development in preterm infants and to investigate the possible association between BPD severity and preeclampsia.

Methods The study group involved preterm infants ( $\leq 32$ gestational week) born to a preeclamptic mother with no co-existing medical condition, whereas the comparison group involved preterm infants born to a normotensive mother. BPD was defined as requirement for supplemental oxygen for the first 28 day of life. It was classified as mild, moderate and severe BPD. The demographics and clinical data of the infants were recorded.

Results There were 117 and 215 premature infants in the study and control groups, respectively. The incidence of BPD in preterm infants in the study group (38.5\%) was significantly higher than the control group (19.5\%). The frequency of moderate and severe BPD were significantly higher in the study group. In logistic regression model, preeclampsia was found to be predictive of BPD.

Conclusions Preeclampsia was found to be an important risk factor for BPD development in preterm infants. The incidence of both moderate and severe BPD was significantly higher in preeclamptic mother infants. These findings might be associated with altered angiogenesis in the preeclamptic mother which might be shared with the fetus.

\section{OXYGEN DEPENDENCE IS ASSOCIATED WITH SUPPLY PROTEIN OFFERS AT FIRST 35 DAYS OF LIFE FOR PRETERM INFANTS WITH BRONCHOPULMONARY DYSPLASIA}

doi:10.1136/archdischild-2012-302724.0603

A Moraes-Barbosa, H Pacheco. Pediatrics, Federal Fluminense University, Niteroi, Brazil

Background and Aims The aims of this study was to determine if the time of introduction and diary quantity of amino acid offered at first 35 days of life for newborns with BPD, weight $<1500 \mathrm{~g}$ at birth, may influence the oxygen dependence at final period.

Methods Newborns with BPD, consecutively observed in the NICU (UFF - RJ - Brazil), from April 2006 to March 2008, were divided in Early Start (ES) and Late Start (LE) Groups when they began to received amino acids (AA) up $(n=56)$ or after $(n=48) 72$ hours of life. It was also divided according to the quantity of AA administered in the first week of life $(1.11 \pm 0.04 \mathrm{~g} / \mathrm{kg} /$ day): Larger Supply (LAS) $(n=42)$ and Lower Supply (LOS) $(n=58)$ received a quantity of AA 2 SD above or below this mean.

Results A total of 104 newborns were evaluated. At $35^{\text {th }}$ of life the babies of ES and LE were weighing $34.1 \%$ and $21.9 \%$ more than his weight at birth, respectively, and from LOS and LAS, 39\% and $23.7 \%$ regarding his birth ( $p>0.05)$. At the end of the studied period, $8.9 \%$ of the babies of the ES and $16.7 \%$ of the LE still stayed receiving some oxygen. In LAS $4.7 \%$ and $21 \%$ in LOS were receiving some oxygen support $(p=0.004)$.

Conclusion Nutritional state is decisive in the oxygen necessity at neonatal final period, and the major offers of protein is a priority way.

604 URINARY NGAL (UNGAL) AT BIRTH IS RELATED TO
BRONCHOPULMONARY DYSPLASIA IN PRETERM INFANTS
'S Vandini, 'G Aquilano, ${ }^{2} \mathrm{C}$ Capelli, ' $\mathrm{LT}$ Corvaglia, 'S Galletti, ${ }^{2} \mathrm{G}$ La Manna. 'Neonatology S. Orsola-Malpighi Hospital; ' $N$ ephrology S. Orsola-Malpighi Hospital, University of Bologna, Bologna, Italy

Background and Aims Bronchopulmonary dysplasia (BPD) is a chronic lung disease associated with premature birth and early lung injury. The pathogenesis is multifactorial, including fluid and electrolytes balance that is dependent to renal development during the first weeks of life.

We previously found a correlation between renal development during the first weeks of life and urinary neutrophil gelatinase-associated lipocalin (UNGAL) at birth in very low birth weight infants (VLBW). The aim of this study was to examine the relationship between urinary (UNGAL) and serum NGAL (SNGAL) at birth and BPD.

Methods UNGAL and SNGAL were determined at birth in VLBW. BPD was defined as oxygen need at 36 week gestational age (GA). Statistical analysis was performed with chi square.

Results 44 VLBW admitted at birth in our NICU were included in the study; 2 of them died during stay in NICU. 20/42 infants developed BPD: all were born at $\leq 29$ week (GA) and 14 of them needed diuretics. High values of UNGAL (>100 ng/ml) were observed more frequently among BPD treated with diuretics infants than in the other subjects $(57 \%$ vs $28 \%$, p=0.04).

High levels of SNGAL (>150 ng/ml) were not significantly more frequent in VLBW with BPD.

Conclusions These preliminary data show that high UNGAL at birth is a marker of impaired renal development and fluid balance in preterm newborns, that determine increased lung water and consequently contribute to BPD development.

\section{5 "LOW DOSE" POSTNATAL CORTICOSTEROIDS FOR INFANTS AT RISK OF SEVERE BRONCHOPULMONARY DYSPLASIA}

doi:10.1136/archdischild-2012-302724.0605

${ }^{1,2}$ C Pitan. 'Neonatology, Royal Women's Hospital, Melbourne, VIC, Australia; 2Paediatrics, University College Dublin, Dublin, Ireland

Background Postnatal corticosteroids (PCS) improve short term lung function but may increase the risk of disability in later life. The Dexamethasone: A Randomised Trial (DART) study was designed to address this risk using a 10 day tapering regimen with a total dose of 890 micrograms $/ \mathrm{kg}$. The Royal Women's Hospital, Melbourne selects patients at high risk of bronchopulmonary dysplasia (BPD) to receive steroids as per the DART protocol. The primary aim of this study was to audit the prescribing practices of the DART protocol with a secondary aims of reporting patient outcomes.

Method Patients were identified from an electronic database between January 2006 and December 2009 and medical records reviewed. Infants prescribed PCS other than as per the DART protocol and for preventing BPD were excluded.

Results Forty six infants with mean (SD) gestational age of 25.0 (1.3) weeks and birth weight of 685 (192) g were prescribed dexamethasone. Median (range) duration of therapy was 20 (3-86) days with a median total dexamethasone dose of 1437 (375-9100) micrograms $/ \mathrm{kg}$. There were significant changes on day 3 of treatment in mean airway pressure (MAP) or positive end expiratory pressure (PEEP), $\mathrm{p}<0.001$ and percentage inspired oxygen $\left(\mathrm{FiO}_{2}\right), \mathrm{p}<0.001$. There were seven deaths, with the combined outcome of either death or BPD occurring in 41 (89\%) infants.

Conclusions In our audit prescribing practices deviating outside of hospital protocol, resulting in doses exceeding those recommended. Clinical practice review of PCS therapy in preterm infants is encouraged.

606 DIURETIC USE IN NEONATAL CHRONIC LUNG DISEASE IN ENGLAND 